\title{
Visuomotor Control Post Stroke Can be Affected by a History of Visuospatial Neglect
}

\author{
Jessica Berard ${ }^{1,2 *}$, Joyce Fung ${ }^{1,2}$ and Anouk Lamontagne ${ }^{1,2}$
}

${ }^{1}$ School of Physical and Occupational Therapy, McGill University, Montreal, Canada

${ }^{2}$ Feil \& Oberfeld Research Centre, Jewish Rehabilitation Hospital, Research site of the Montreal Center for Interdisciplinary Research in Rehabilitation (CRIR), Laval, Quebec, Canada

\begin{abstract}
A frequent consequence of stroke is locomotor disturbance. Locomotor steering, which relies heavily on the use of visual information, can be affected by a stroke but little is known about such disturbance. We investigated the ability of nine chronic stroke patients to steer in response to changes in the optic flow in a single-subject design. Subjects were instructed to walk straight for $5 \mathrm{~m}$ in a virtual environment with or without a perturbation of the focus of expansion (FOE), rotating horizontally $40^{\circ}$ towards the right or left from the midline. Subjects exhibited 3 distinct behaviours: (I) $4 / 9$ subjects showed no deficits in their locomotor strategy and responded by rotating their head and body in the direction opposite to the FOE shift, as previously reported in healthy individuals: (II) 2/9 subjects showed some degree of steering adjustments in response to the flow but resulted in slightly higher net heading errors; (III) 3/9 showed large net heading errors that were especially pronounced when the FOE was rotated in the contralesional direction. The most important distinction between subjects who performed normally and those who had deficits was that the latter had a history of visuospatial neglect, suggesting that spatial neglect can have a persistent impact on visuomotor control. Our findings also suggest that standard pen-and-paper tests are not sufficient for detecting all forms of neglect and may adequately detect deficits in processing of dynamic visual motion or for stimuli located in far space.
\end{abstract}

Keywords: Stroke; Neglect; Optic flow; Walking; Heading; Cerebrovascular accident

\section{Introduction}

Real world navigation is guided by visual information that enables the locomotor pattern to be adjusted in accordance to environmental constraints and to the intended goal. Optic flow, which is a pattern of visual motion at the retina, can provide critical information to the perception and control of heading [1]. When provided with rich visual cues, healthy adults are able to adjust their gait in response to changes in optic flow [2,3], even with old age [4]. It has been reported that a staggering $86 \%$ of individuals post stroke have some sort of oculomotor dysfunction [5] that can impact on gaze control [6] and in establishing a stable frame of reference [7] during locomotion. There is also evidence to suggest that optic flow perception can be impaired after stroke. Vaina et al. [8] reported that stroke patients with occipital-parietal or rostral-dorsal-parietal lesions had deficits for visual motion processing. Others [9] showed that approximately $50 \%$ of the stroke patients tested had some level of motion processing deficit, Interestingly, only one of the 21 subjects tested reported higher discrimination thresholds for radial flow direction, the type of flow pattern experienced while walking. While psychophysical evidence indicates that visual motion processing impairments can be present after stroke, it is unclear how these deficits translate into locomotor impairments. Our previous research has shown that stroke patients were not able to automatically adjust their walking speed in response to changing speeds of visual flow [10]. More recently, we have shown that some people after stroke have difficulty responding to translations of optic flow cues [11]. In this single-subject design study, we investigated the ability of 9 individual chronic stroke patients to steer their walking when exposed to a horizontal rotation of optic flow focus of expansion (FOE). Possible determinants of visuomotor control deficits were examined using cognitive, visuospatial and functional gait measures as well as by analyzing the history and location of the stroke.

\section{Methods}

\section{Subjects}

Nine patients with stroke participated in the study (Table 1). The subjects tested ranged in ages from 41-71 years and comprised of six men and three women. Four of the patients tested had experienced hemorrhagic strokes, while the other 5 patients had had ischemic strokes. The patients recruited had a variety of confirmed lesion locations, including cortical regions supplied by the middle cerebral artery and sub-cortical structures such as the thalamus and basal ganglia. The inclusion criteria for the study were: 1) the ability to walk independently for more than 10 meters, with or without a walking aid; 2) absence of dementia or cognitive impairments (scoring $\geq$ 27 on the Mini-Mental State Examination [12]); 3) residual motor deficits of the lower limb ( $\leq 6$ on the Chedoke-McMaster impairment scale [13] for both the paretic leg and foot; 4) normal or corrected to normal visual acuity $(\geq 20 / 40)$. We also ensured that the patients were currently not demonstrate any evidence of visuospatial neglect as indicated by a normal performance on two pen-and-paper neglect tests: the star cancelation and line bisection tasks [13,14]. Despite not currently exhibiting spatial neglect, five of the subjects had a clinical history of visual neglect. In order to characterize the participants,

*Corresponding author: Jessica Berard, Feil/Oberfeld/CRIR Research Center, Jewish Rehabilitation Hospital, 3205 Place Alton-Goldbloom, Laval Quebec, Canada, Tel: 450 688-9550 (4823); Fax: 450-688-3673; E-mail: jb3433@columbia.edu

Received October 21, 2011; Accepted February 14, 2012; Published February 19,2012

Citation: Berard J, Fung J, Lamontagne A (2012) Visuomotor Control Post Stroke Can be Affected by a History of Visuospatial Neglect. J Neurol Neurophysiol S8. doi:10.4172/2155-9562.S8-001

Copyright: $(2012$ Berard J, et al. This is an open-access article distributed under the terms of the Creative Commons Attribution License, which permits unrestricted use, distribution, and reproduction in any medium, provided the original author and source are credited. 
we also performed the following assessments: Montreal Cognitive Assessment (MoCA), Trail Making Test A \& B (TMT-AB) and Functional Gait Assessment (FGA). MoCA is a neuro-cognitive test designed to detect mild cognitive impairments. While similar to the MMSE, the MoCA has better sensitivity. Scores $<26$ are suggestive of mild cognitive impairment [15]. TMT is a measure of visual attention, visual scanning, sequencing and cognitive flexibility [16]. We recorded the time to complete part $\mathrm{A}$ and $\mathrm{B}$ and compared them to age-matched norms [17]. The FGA evaluates postural stability during a variety of dynamic gait activities [18]. Lesion location and details concerning the vascular incidents were obtained through the patient's medical record. All subjects consented to their participation in accordance to the declaration of Helsinki and the project was approved by the institutional research ethics board.

\section{Experimental setup}

Subjects walked overground in a large, open-space laboratory ( $12 \mathrm{~m} \times 8 \mathrm{~m}$ walking area) while wearing a helmet-mounted display unit (HMD; NVisor with $60^{\circ}$ diagonal field of view and $1280 \times 1084$ pixels resolution) displaying a virtual room with the same dimensions. Details of the setup and paradigm have been reported previously [4]. Subjects were outfitted with 39 passive reflective markers on anatomical landmarks as defined in the Vicon Plug-In-Gait model. Kinematic data were captured at $120 \mathrm{~Hz}$ with a 12 -camera Vicon-512 $2^{\text {ma }}$ system. Movements of the head were tracked in real-time via three markers placed on the HMD and used by the CAREN-3 system (Computer Assisted Rehabilitation Environments, MOTEK BV) to update the subject's position and orientation in the virtual scene. As such, movements of the head were synchronized and displayed in the HMD in real time, with a negligible delay of $25 \mathrm{~ms}$.

\section{Protocol}

Prior to any data collection, subjects were given the opportunity to walk in the virtual environment to habituate to the task. All subjects indicated that they were comfortable in the virtual environment and appeared to walk similarly to their normal behaviour in the physical environment. Walking data were collected in four phases. We used a single-subject design of $A_{1}-B_{1}-A_{2}-B_{2}$ such that each participant received the same order of stimulus manipulation. The A phases were to collect a stable baseline and to measure the subjects' performance without any perturbation of the flow. Subjects were instructed to "walk straight in the virtual world", i.e. walking straight with respect to the scene that was displayed in the HMD. Since there was no visual perturbation in phase A, this corresponded to simply walking straight for 5 meters. In the B phases, subjects were again instructed "to walk straight in the virtual world", but after they had walked $1.5 \mathrm{~m}$, the scene would gradually rotate to the right or the left so that the total rotation was $40^{\circ}$ to the left or right over the remaining 3.5 meters of forward displacement. This type of visual disturbance is very similar to what one might experience if they where to make a head or eye rotation to the left or right while walking forward with the head straight. In the first perturbation phase $\left(\mathrm{B}_{1}\right)$ the FOE was rotated towards the paretic (contra-lesional) side and in the second perturbation phase $\left(\mathrm{B}_{2}\right)$ the FOE was rotated toward the non-paretic (ipsi-lesional) side. Each subject completed 3-7 trials per phase, depending on their endurance and level of fatigue. Subjects took mandatory breaks between blocks of trials, but were also permitted to rest at any point if they felt fatigued. At all times, a therapist followed close to the patient should they become unstable or need assistance during walking. No falls or near-falls were observed.

\section{Data analysis}

Head orientation and body's centre of mass $(\mathrm{CoM})$ positions were

\begin{tabular}{|c|c|c|c|c|c|c|c|c|c|c|c|c|c|c|}
\hline Subject & Gender & $\begin{array}{l}\text { Time } \\
\text { Since } \\
\text { Stroke } \\
\text { (yrs) }\end{array}$ & $\begin{array}{l}\text { Age } \\
\text { (yrs) }\end{array}$ & $\begin{array}{l}\text { Side of } \\
\text { Lesion }\end{array}$ & Etiology & Lesion Location & $\begin{array}{l}\text { FGA }^{a} \\
(/ 30)\end{array}$ & $\begin{array}{l}\mathrm{CM}^{\mathrm{b}} \\
\text { foot, } \\
\text { leg } \\
(/ 7)\end{array}$ & $\begin{array}{c}\text { MoCA }^{c} \\
(/ 30)\end{array}$ & $\begin{array}{c}\text { MMSE } \\
(/ 30)\end{array}$ & $\begin{array}{c}\text { Trail A/B } \\
\text { (s) }\end{array}$ & $\begin{array}{l}\text { History of } \\
\text { Neglect }\end{array}$ & $\begin{array}{l}\text { Gait } \\
\text { Speed }^{d} \\
(\mathrm{~m} / \mathrm{s})\end{array}$ & Type \\
\hline S1 & $M$ & 1.2 & 58 & $\mathrm{~L}$ & Ischemia & $\begin{array}{l}\text { Thalamus and internal } \\
\text { capsule at the temporo- } \\
\text { occipital junction }\end{array}$ & 23 & 6,6 & 22 & 29 & $60 / 246$ & No & 0.75 & 1 \\
\hline $\mathrm{S} 2$ & $M$ & 1.7 & 72 & $\mathrm{R}$ & Hemorrhage & $\begin{array}{l}\text { Thalamus and right internal } \\
\text { capsule }\end{array}$ & 30 & 6,6 & 23 & 30 & $56 / 162$ & No & 0.62 & 1 \\
\hline S3 & $M$ & 3.7 & 41 & $\mathrm{R}$ & Ischemia & $\begin{array}{l}\text { Basal ganglia and parietal } \\
\text { cortex }\end{array}$ & 24 & 3,6 & 24 & 30 & $27 / 120$ & Yes & 0.66 & 2 \\
\hline S4 & $M$ & 0.5 & 57 & $\mathrm{R}$ & Hemorrhage & Thalamus & 15 & 3,3 & 26 & 30 & $29 / 44$ & No & 0.27 & 1 \\
\hline S5 & $M$ & 3.1 & 42 & $\mathrm{R}$ & Hemorrhage & Basal ganglia & 24 & 3,5 & 19 & 30 & $85 / 330$ & Yes & 0.55 & 3 \\
\hline S6 & $\mathrm{F}$ & 15.3 & 43 & $\mathrm{R}$ & Ischemia & $\begin{array}{c}\text { Middle cerebral artery } \\
\text { territory }\end{array}$ & 25 & 3,5 & 24 & 29 & $25 / 55$ & Yes & 0.79 & 3 \\
\hline S7 & $M$ & 6.7 & 64 & L & Hemorrhage & $\begin{array}{c}\text { Sylvian fissure and lentiform } \\
\text { nucleus }\end{array}$ & 19 & 5,6 & 23 & 29 & $32 / 157$ & Yes & 0.28 & 3 \\
\hline S8 & $\mathrm{F}$ & 2.1 & 71 & L & Ischemia & $\begin{array}{l}\text { Corona radiata and frontal } \\
\text { lobe }\end{array}$ & 16 & 3,5 & 29 & 29 & $64 / 320$ & No & 0.50 & 1 \\
\hline s9 & $\mathrm{F}$ & 0.8 & 51 & $\mathrm{R}$ & Ischemia & Sylvian fissure & 15 & 3,5 & 29 & 29 & $50 / 120$ & Yes & 0.35 & 2 \\
\hline
\end{tabular}

aFunctional Gait Assessment

${ }^{b}$ Chedoke-McMaster

"Montreal Cognitive Assessmen

${ }^{\mathrm{d} A}$ verage of actual walking speeds during testing

Table 1: Characteristics of participants. 
Citation: Berard J, Fung J, Lamontagne A (2012) Visuomotor Control Post Stroke Can be Affected by a History of Visuospatial Neglect. J Neurol Neurophysiol S8. doi:10.4172/2155-9562.S8-001

calculated in three dimensions with the Vicon Plug-In-Gait model using marker positions and anthropometric measurements. Data were exported to Matlab and low-pass filtered at $10 \mathrm{~Hz}$ (Butterworth dual-pass filter). In this study we included people with either right or left cortical strokes. By convention, the polarity of the data was adjusted such that a leftward rotating FOE was towards the paretic side and a FOE rotating to the right was towards the non-paretic for all participants.

\section{Outcome variables}

The scene as viewed in the HMD is a function of head rotation and heading, the latter being the angular displacement of the CoM trajectory

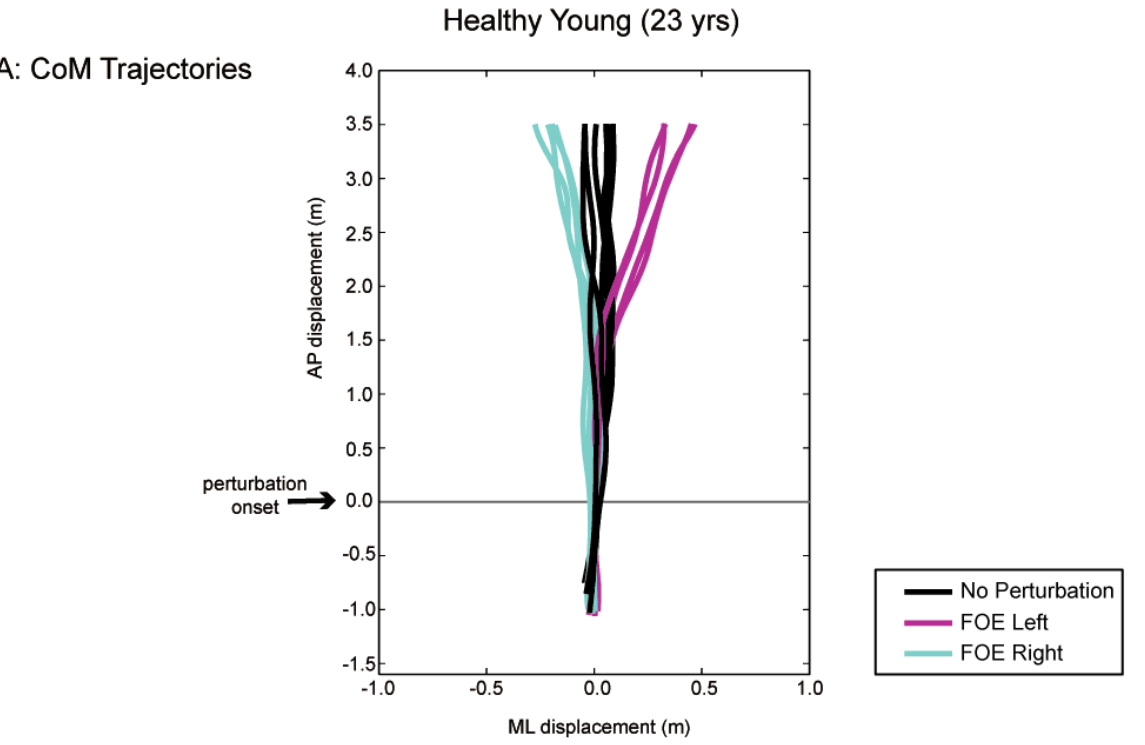

B: Net Heading Correction

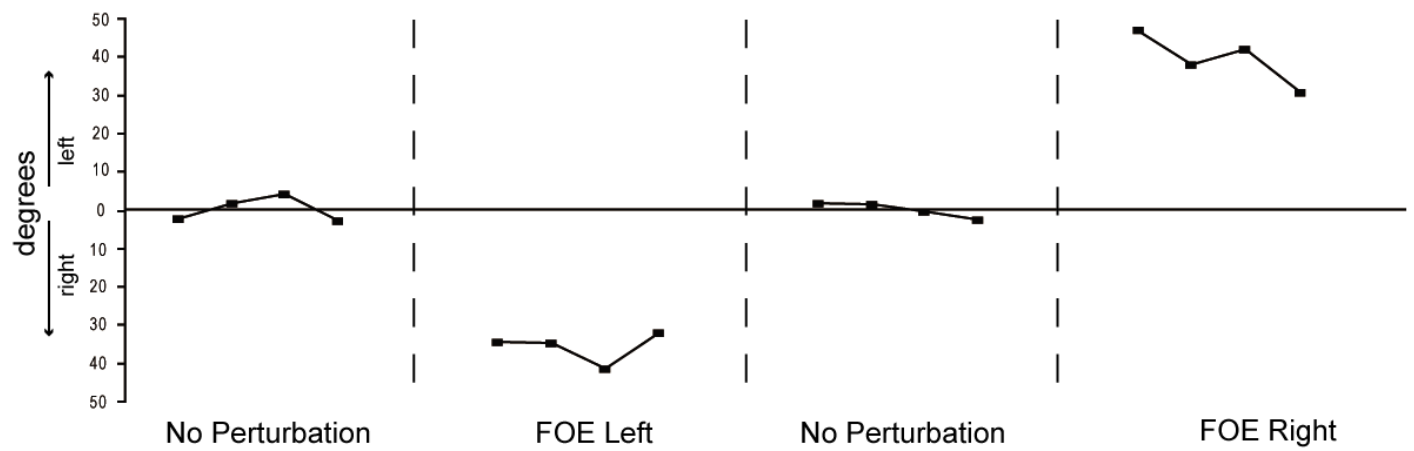

C: Net Heading Error

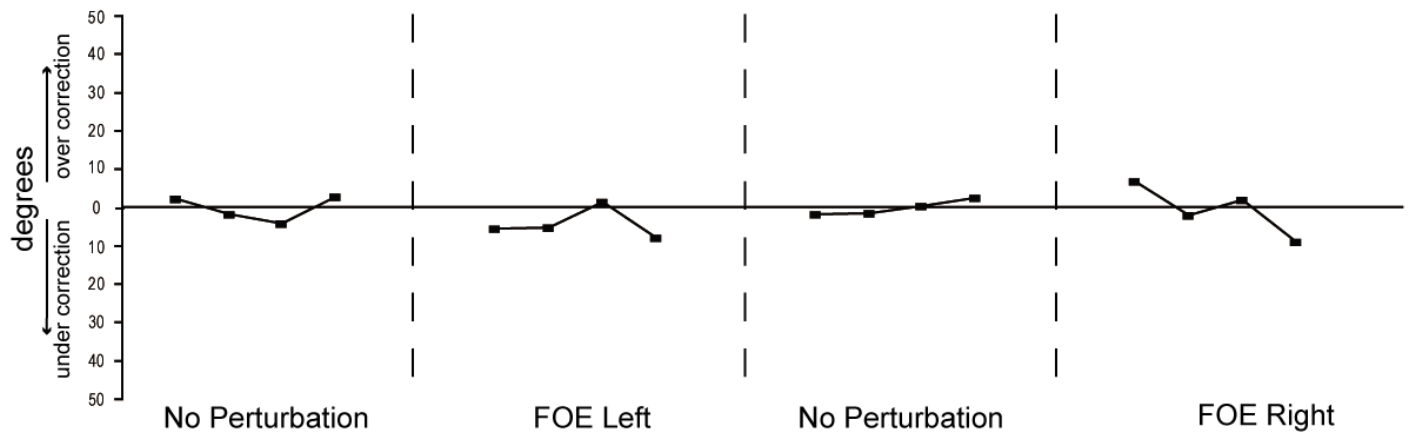

Figure 1: Representative data from a healthy young subject (age 23): A) Sample trials of CoM displacements under different FOE conditions. B) Net heading correction at the end of the walking trial. Each point indicates the magnitude of reoriention in the physical world at the end of the trial (when the FOE was rotated $40^{\circ}$ for perturbation conditions). Data are plotted sequentially in the order of data collection, grouped by perturbation condition. C) Heading error, i.e. the degrees (virtually) off from the midline of the room at the end of the trial. 
Citation: Berard J, Fung J, Lamontagne A (2012) Visuomotor Control Post Stroke Can be Affected by a History of Visuospatial Neglect. J Neurol Neurophysiol S8. doi:10.4172/2155-9562.S8-001

from the initial position in the horizontal plane (or from the onset of optic flow perturbation in the B phases). The combined response of head rotation plus heading most accurately reflects the camera view of the scene in the HMD. Therefore, steering performance at the end of the trial was assessed using a 'net heading correction' variable based on the sum of head rotation and heading. We also calculated the error of performance by subtracting the magnitude of perturbation from the net heading correction. An over-correction is depicted by a positive error, i.e. the participant adjusted by more than $40^{\circ}$. Likewise, undercorrection is depicted by a negative error, i.e. failing to reach $40^{\circ}$ of net heading correction. Head angle, heading and net heading correction data at $5 \mathrm{~m}$ of forward walking ( $3.5 \mathrm{~m}$ after perturbation onset) were averaged separately for each A and B phase.

\section{Statistical analysis}

The data for each subject were examined by visual inspection and quantitative comparisons with non-parametric statistical analyses. Kruskal-Wallis tests were used to detect any differences between optic flow conditions. Where significant effects existed, Mann-Whitney $\mathrm{U}$ tests with Bonferroni correction were used to identify differences between conditions. Statistics were calculated using PASW Statistic 18. Significance level was set to $\mathrm{p}=0.05$.

\section{Results}

Before the behaviour of stroke subjects can be described, it is useful to understand the healthy subjects' response to rotational flow. When exposed to a rotation of the FOE, healthy subjects adjust their walking trajectory in a direction opposite to the FOE perturbation (Figure 1A). When the FOE is rotated towards the right, the walking trajectory is adjusted in the physical environment towards the left. This CoM displacement contributes to a shift towards the midline, perceived as staying straight in the virtual environment, as viewed in the HMD. Subjects also compensate by rotating their head opposite to FOE direction (not shown), which is in the same direction as the physical trajectory adjustment. The combined sum of head angle and CoM angular displacement (heading) gives the net heading correction in the HMD. Figure 1B shows the net correction of each trial for the sample data participant. When the FOE is rotating left, the subject responds with approximately $40^{\circ}$ of net heading correction to the right and vice versa for the other direction of rotation. The subject can thus
Type 1
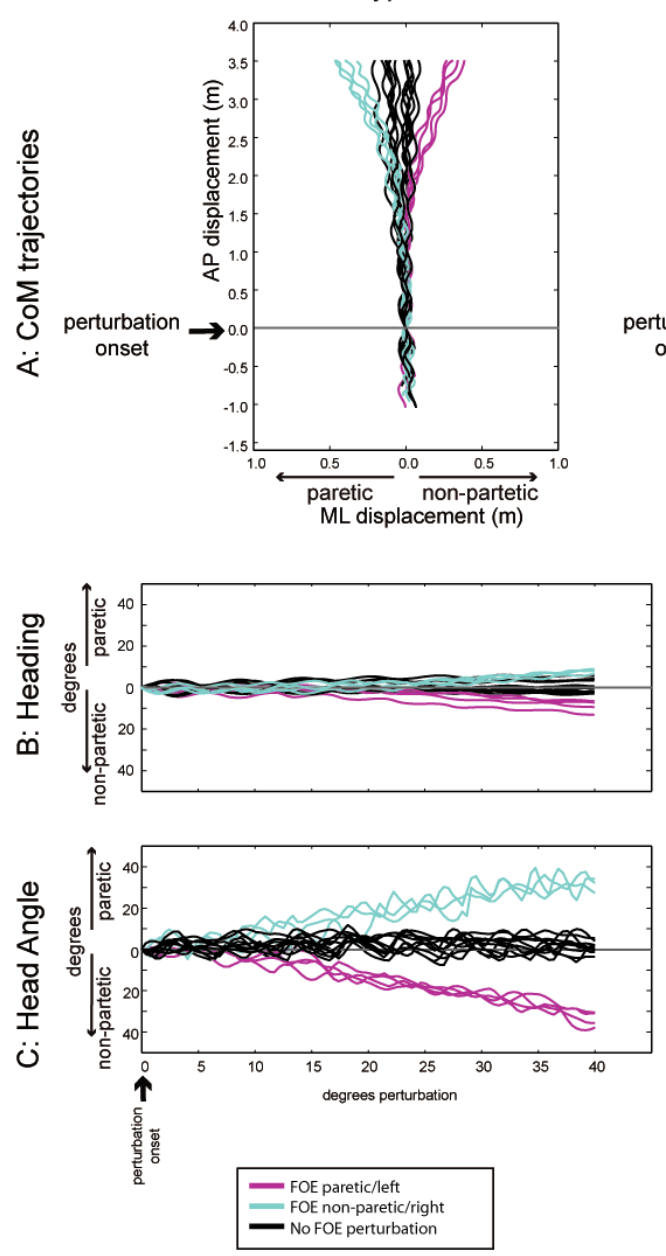

Type 2
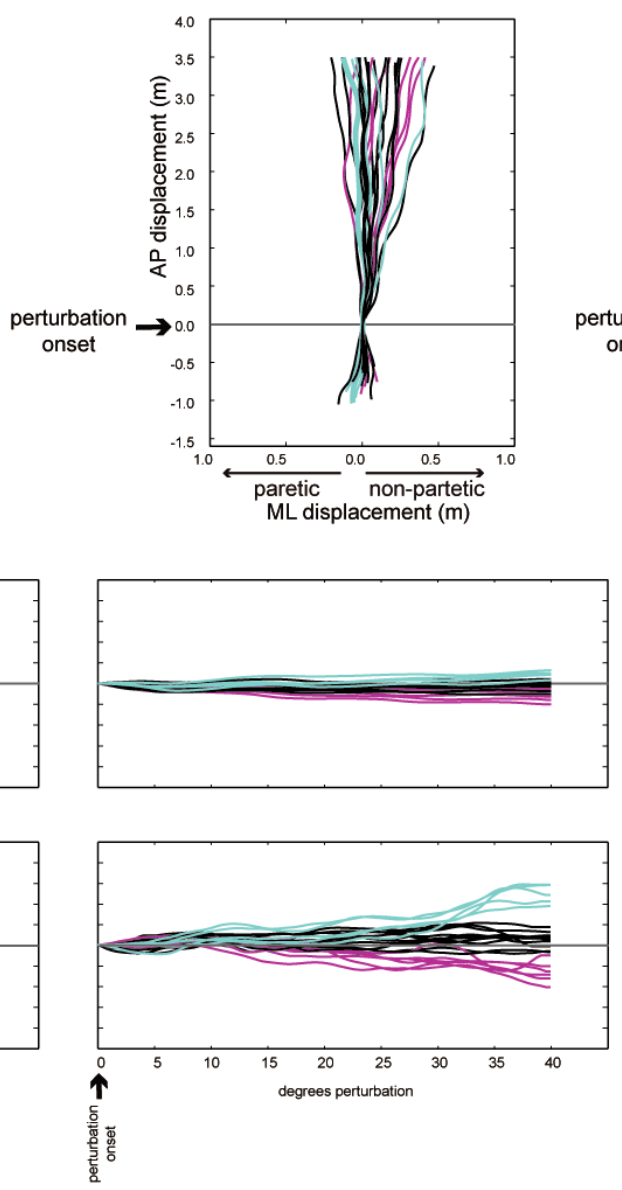

Type 3
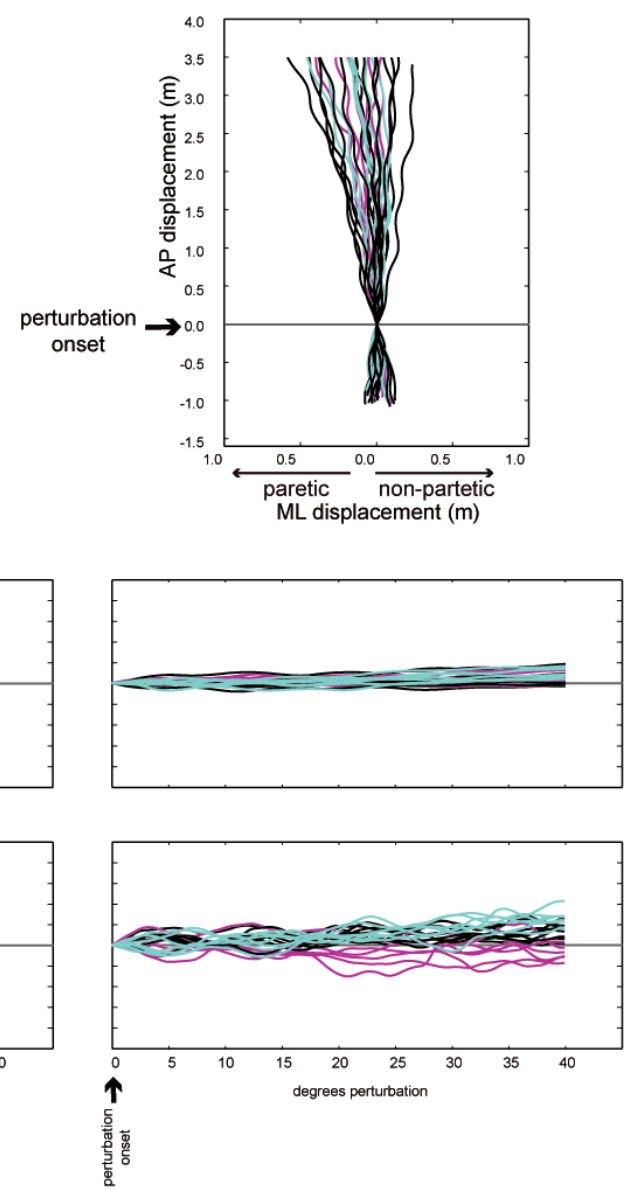

Figure 2: CoM trajectories for 3 participants (S4=type 1, S3=type 2, S5=type 3), each displaying typical profiles for their respective classification. Type-1 patients typically responded similarly to healthy young subjects. Type-2 and -3 patients showed irregular CoM trajectories. B) Angular displacement of the CoM (heading) as a function of forward displacement. C) Head yaw as a function of forward displacement. Type-1 patients show large reorientations of the head in the direction opposite to the FOE rotation. Type-2 patients show smaller head rotations in the direction opposite to the FOE. Type-3 show little or no head reorientations. 
Citation: Berard J, Fung J, Lamontagne A (2012) Visuomotor Control Post Stroke Can be Affected by a History of Visuospatial Neglect. J Neurol Neurophysiol S8. doi:10.4172/2155-9562.S8-001

correct fully for the perturbations, resulting in small net heading errors in all conditions (Figure 1C). The stroke patients showed a variety of responses, which were classified into three distinct types, based on visual inspection and quantitative comparisons. Figure 2 shows traces of the CoM, heading and head angle for representative individuals from each type. The first type of behaviour (type 1) resembled that of healthy individuals and was exhibited by 4 subjects (S1,S2,S4,S8). Their CoM displacements showed the typical v-shaped pattern of CoM trajectories with the CoM orienting away from the FOE shift in the perturbation conditions (Figure 2A). Accordingly, small angular displacements of the CoM could be observed in the same direction as the linear displacement
(Figure 2B). These individuals also showed very large head rotations in directions opposite to the FOE rotations (Figure 2C). Heading and head yaw data were summed to compute the net heading correction, which revealed how much the participant corrected in the virtual environment (Figure 3A). These type 1 individuals displayed very small errors (Figure $3 \mathrm{~B}$ ) in each phase, generally less than $10^{\circ}$. Table 2 shows the mean net heading error of all participants. There were no significant differences in net heading error between the phases with or without perturbations for any individuals classified as type 1 . Two individuals exhibited mild to moderate impairments in heading corrections and were classified as type $2(\mathrm{~S} 3, \mathrm{~S} 9)$. They did make locomotor adjustments

Comparison BetweenTypes

for Net Heading

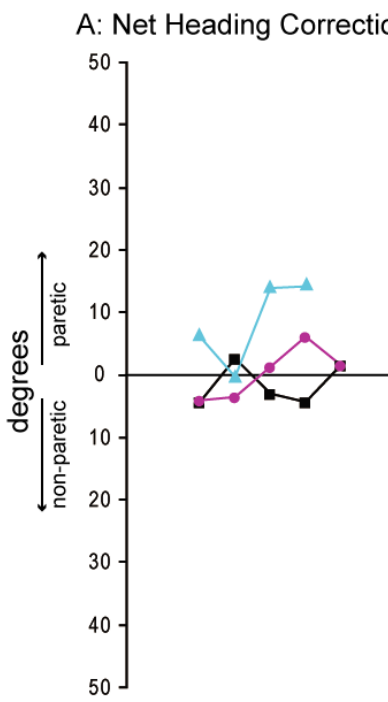

No Perturbation

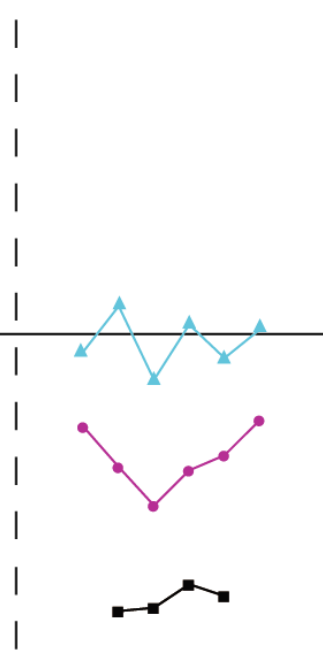

FOE Paretic

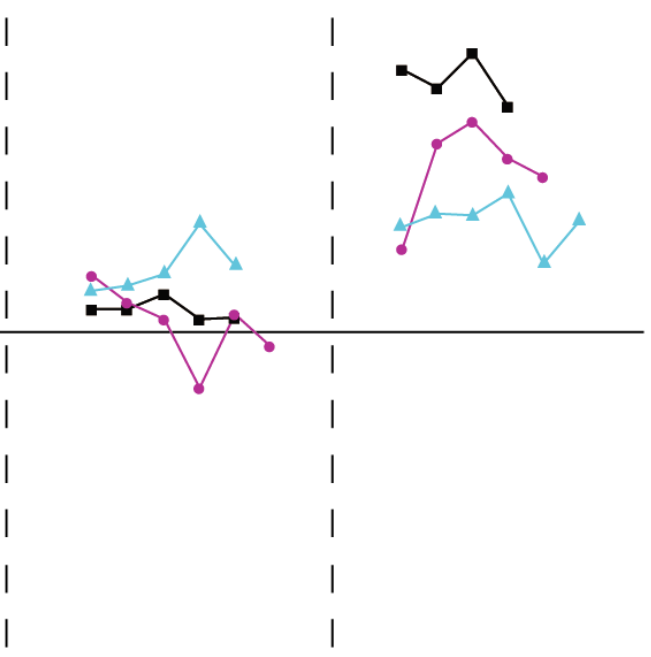

No Perturbation

FOE Non-Paretic

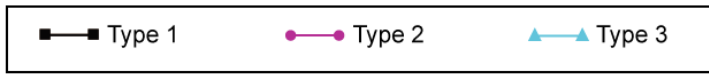

\section{B: Net Heading Error}

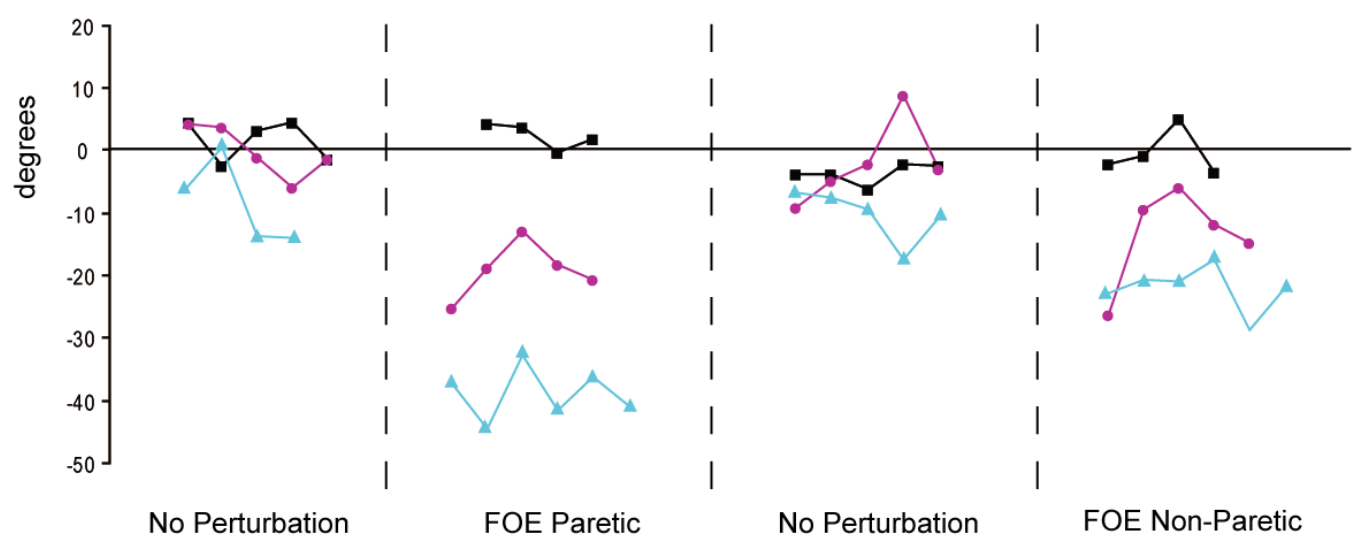

Figure 3: A) Net correction of representative subjects from each of the three groups of participant. B) Net errors from representative subjects. 
Citation: Berard J, Fung J, Lamontagne A (2012) Visuomotor Control Post Stroke Can be Affected by a History of Visuospatial Neglect. J Neurol Neurophysiol S8. doi:10.4172/2155-9562.S8-001

Page 6 of 8

\begin{tabular}{|c|c|c|c|c|c|c|}
\hline Subject & $\begin{array}{c}\text { No Perturbation } 1 \\
\text { Mean } \pm \text { SD }\end{array}$ & $\begin{array}{l}\text { FOE Paretic } \\
\text { Mean } \pm \text { SD }\end{array}$ & $\begin{array}{c}\text { No Perturbation } 1 \\
\text { Mean } \pm \text { SD }\end{array}$ & $\begin{array}{l}\text { FOE Non-Paretic } \\
\text { Mean } \pm \text { SD }\end{array}$ & $\begin{array}{l}\text { Visual Inspection of } \\
\text { Performance }\end{array}$ & Type \\
\hline $\mathrm{S} 1$ & $0.7 \pm 6.7$ & $-7.6 \pm 4.3$ & $-2.5 \pm 4.1$ & $-1.7 \pm 5.8$ & none & 1 \\
\hline $\mathrm{S} 2$ & $-3.8 \pm 8$ & $-8.5 \pm 7.2$ & $-3.2 \pm 7.3$ & $5.6 \pm 3.8$ & none & 1 \\
\hline S3 & $-0.2 \pm 4.2$ & $-20.4 \pm 5.0^{*}$ & $-1.4 \pm 6.2$ & $-13.7 \pm 7.8^{*}$ & mild to moderate & 2 \\
\hline S4 & $1.6 \pm 3.4$ & $2.3 \pm 2.0$ & $-3.8 \pm 1.5$ & $-0.5 \pm 3.8$ & mild to moderate & 1 \\
\hline S5 & $-8.4 \pm 6.9$ & $-39.0 \pm 4.2^{* \perp}$ & $-10.5 \pm 4.2$ & $-21.4 \pm 4.0^{*} \perp$ & moderate to severe & 3 \\
\hline S6 & $-10.7 \pm 7.2$ & $-31.2 \pm 6.4^{* \perp}$ & $-6.7 \pm 5.2$ & $-21.9 \pm 1.8^{* \perp}$ & moderate to severe & 3 \\
\hline S7 & $-1.9 \pm 6.4$ & $-37.7 \pm 8.5^{*} \perp$ & $-3.0 \pm 8.2$ & $-7.1 \pm 1.7^{\star \perp}$ & moderate to severe & 3 \\
\hline S8 & $2.6 \pm 1.9$ & $-5.47 \pm 2.7$ & $-1.5 \pm 3.9$ & $-7.0 \pm 2.5$ & none & 1 \\
\hline S9 & $-4.79 \pm 1.9$ & $-10.49 \pm 3.27^{*}$ & $-0.47 \pm 3.9$ & $-18.52 \pm 6.65^{*}$ & mild to moderate & 2 \\
\hline
\end{tabular}

*denotes significant difference from no perturbation conditions

${ }^{\perp}$ denotes significant difference between paretic and non-paretic sides

type 1: normal Response

type 2: mild to moderate impairments

type 3 moderate to severe asymmetric responses

Table 2: Average Net Heading Responses at the end of the trial for each subject in each phase of testing. Negative values indicate undershooting.

in response to the flows presented, but they were not able to fully correct for the perturbation, resulting in a marked under-correction of net heading. The CoM trajectories for these subjects did not follow the typical pattern shown by healthy subjects and were variable in terms of the magnitude and direction of CoM displacements that were also slightly asymmetrical (Figure 2A). These individuals did respond with a head rotation opposite to the flow direction (Figure 2C), but the magnitude was smaller than that shown by type 1 individuals, resulting in a markedly smaller total net heading correction (Figure $3 \mathrm{~A}$ ). The errors were significantly higher in the perturbation conditions as compared to those in the baseline, ranging from $10^{\circ}$ to $20^{\circ}$ of undercorrection. There were no statistical differences in net heading error between FOE shifts to the paretic or non-paretic side (Table 2). Three individuals exhibited moderate to severe impairments in heading correction $(\mathrm{S} 5, \mathrm{~S} 6, \mathrm{~S} 7)$. The CoM trajectory direction and deviations were generally inconsistent and highly variable, even when no perturbation occurred. The CoM adjustments were asymmetric, with smaller or misaligned headings when the FOE was rotating towards the paretic or contra-lesional side (Figure 2B). The head rotations were notably smaller for this third group (Figure 2C). The net heading responses for these individuals were also asymmetrical. There were virtually no net heading corrections when the FOE was rotating towards the paretic side and only moderate responses when the FOE was rotating towards the non-paretic side (Figure 3). The heading errors displayed this asymmetry, with very large errors in the $30^{\circ}-40^{\circ}$ range when the FOE was rotating toward the paretic or contra-lesional side. The errors for the ipsi-lesional rotations of the FOE were mild to moderate, ranging from $7^{\circ}$ to $22^{\circ}$. Evidence for asymmetry was also supported by the net heading errors that were significantly larger for contra-lesional FOE rotations as compared to ipsi-lesional ones (Table 2). Individual net headings, errors and CoM trajectories for each subject are included in the supplementary material.

\section{Discussion}

Using optic flow to guide heading is central to locomotor adaptation and steering behaviour. In the present study, visually guided steering is disrupted after stroke in some but not all subjects. Considering the heterogeneous nature of stroke and multifaceted interactions of an individual with the environment and task goals, it is not surprising to find variable responses. Almost half (4/9) of our participants performed the task very well, exhibiting small errors and resembling the typical response trajectories seen in healthy individuals, whereas the others (5/9) showed large heading errors and a wide range of CoM deviations.

\section{Performance related to psychometrics}

The cognitive function of processing speed and visuomotor skills was generally impaired, as revealed by the results of the psychometric tests, but the severity of the impairment did not reliably predict the subject's performance during the walking task. Seven of the nine subjects fell below the $10^{\text {th }}$ percentile for their age group in the time required to complete Trail Making Test (TMT) B, including all 4 individuals in the normal response group (type 1). The two who did not show major impairments in the TMT were S4 (type 1; $90^{\text {th }}$ percentile) and $S 7$ (type $3 ; 60^{\text {th }}$ percentile). This is surprising, as S7 had the second largest errors when walking with a contralesional FOE rotation and yet obtained the second best score for TMTB among all subjects. The MoCA scores were 26 or less for all but two subjects (S8 and S9), suggesting the presence of some cognitive impairment in the other 7 individuals [15]. The worst score belonged to S5 (Type 3) who obtained only 19 on the MoCA and the same individual also had the highest error scores overall (Table 2). However, other than this extreme case, there seems to be little or no association between MoCA scores and net correction errors. For instance, the second lowest score on the MoCA was S1 with a score of 22, who performed normally and was categorized as type 1 in their steering response; and $S 4$ who might be 
considered one of the better performers with very small errors had a mildly impaired score of 26. The results from the psychometric tests we performed in this study would clearly indicate the general presence of mild cognitive impairment post stroke, but given the distribution of scores across individuals and steering behaviour types, it does not appear that there is any direct association between these assessments and the ability to use optic flow for locomotor adjustments.

\section{Performance related to physical impairments}

Individual steering performance does not appear to relate to the underlying motor impairments, such as motor recovery post stroke, slow gait speed or impaired gait functions. In each type of steering response, there was a mix of high and low functioning individuals, as reflected by the FGA and Chedoke McMaster Stroke Assessment scores. If the ability to reorient in response to optic flow was limited solely by motor and biomechanical factors, we would expect the worst performance from our most impaired subject. S4 was one of the most severely affected subjects in terms of motor function and gait ability (lowest FGA score and slowest walking speed) and yet performed virtually identical to healthy subjects in terms of the steering task. Similarly, S6 (type 3), who had very mild locomotor impairments and had the second highest FGA score, showed large heading errors while steering. It is clear from our data that motor or gait impairment alone cannot explain altered steering strategies and that other factors contributed.

\section{Visuospatial neglect}

As discussed above, it is evident that the performance differences between groups are not related to the general cognitive capacity or mobility of our subjects. However, what could explain our findings are differences in the ability to process and/or integrate visual motion. We excluded subjects with current visuospatial neglect (as screened by the line bisection test and the star cancelation task), but not those with a history of visual neglect. Remarkably, all individuals classified as type 2 or 3 in this study all had a history of visual neglect. This was an unexpected finding, as most of the subjects tested were chronic, living with a stroke for several years. Others have shown that deficits that arise from a damage to visual processing areas can be enduring [19-21]. Our findings seem to support this notion, though the everyday behaviour of these subjects would indicate that they have learned ways to compensate for their underlying deficit. For locomotor tasks, both optic flow and egocentric cues can be used to guide heading $[3,22,23]$. Subjects who have experienced visuospatial neglect may have adapted their locomotor behaviour to selectively use external references as cues (egomotion strategy), while not heavily relying on visual motion.

We believe that this is the first study to show persistent deficits on the steering control of locomotion even after the clinical signs of neglect could no longer be detected. However, it is well known that people with neglect often show altered gait strategies. People with neglect often veer to one side [24-27], though there is some debate as to the direction of bias. Some studies have reported veering towards the ipsilesional or non-paretic side [25,26], whereas others have reported veering on both sides $[27,28]$. As well, the direction of steering appears to be modalityspecific [24] such that it has been shown that some individuals veer to the left when navigating in a wheelchair while one of the same subjects veered to the right while walking. In the present study, two of the subjects with a history of neglect (S5 and S6) showed a veering bias in the no perturbation conditions. Both subjects tended to veer towards the paretic side, even in the absence of any visual manipulation. This is consistent with the observations reported by Tromp [27] where individuals with mild neglect tended to veer in the paretic direction. The association between poor steering performance in the perturbation conditions and previous history of neglect also highlights the problems in proper screening for visuospatial neglect. Conventional paper and pencil tests do not capture the capacity for attending to dynamic visual cues or for far space [29]. Some have reported that neglect is worse for far space than near [30,31]. Recently, others have shown that subjects with a history of neglect have deficiencies in identifying static cues in a $3 \mathrm{D}$ environment, despite having no difficulties with standard penand-paper tests [32]. It seems that for some of subjects had resolved the neglect for personal space but their neglect for far space lingers.

\section{Brain lesions}

The classification of the subjects, which was based on the ability to make heading corrections, could not be related to location or cause (ischemic vs. hemorrhagic) of the lesion. The attempt to characterize individual lesions was performed posthoc by reviewing CT-scan reports provided in the medical charts. Unfortunately, the extent of details provided in the reports was judged not sufficiently detailed to provide a precise picture of the brain structures that were involved in the stroke, nor did they allow us to assess the extent of damage in a particular region. Despite limited specificity, of or lesion data, we were able to make the broad observation that subjects who showed impairments in heading control had lesion sites generally consistent with anatomical correlates of spatial neglect. Others have proposed a perisylvian network including the superior/middle temporal gyri, inferior parietal and ventrolateral frontal cortices which are responsible for spatial orientation [33]. Lesions in these areas may give rise to spatial neglect. Four of the subjects who showed some level of impairment in heading control (type 2 or 3 ) indeed had lesions involved in this perisylvian network (S3, S6, S7 and S9). In addition, it has also been reported recently that lesions of the basal ganglia are associated with spatial neglect, particularly in the chronic phase, which is also consistent with the altered performance of 3 of our subjects (S3, S5, S7) whose lesions were reported to involve structures of the basal ganglia [34].

\section{Limitations}

The purpose of this study was exploratory in nature and we sought to investigate the relationship between stroke and the ability to visually navigate with changing optic flows. While subjects were assessed on various cognitive and visuospatial tasks, perception of visual motion alone was not examined. Thus it cannot be concluded whether the poor heading performances were related to changes in perception of motion or if they were rather the result of altered sensorimotor integration. Moreover, if post-stroke visuospatial neglect of far space is an important factor, as suggested by the present findings, it will be important to investigate the ability to process visual motion in far space as compared to near personal space.

\section{Conclusions}

Results from this study show that the ability to use optic flow during locomotion can be preserved in some individuals after stroke. However, profound steering errors can also be found in certain individuals with a history of visuospatial neglect when exposed to an FOE rotating away from the midline, particularly in the contralesional 
Citation: Berard J, Fung J, Lamontagne A (2012) Visuomotor Control Post Stroke Can be Affected by a History of Visuospatial Neglect. J Neurol Neurophysiol S8. doi:10.4172/2155-9562.S8-001

direction. Persistent visuospatial neglect, especially for far space and/or dynamic visual cues may not be detected through conventional static pen and paper tests and could be an important factor underlying poststroke steering deficits.

\section{Acknowledgments}

This work was supported by the Canadian Institute of Health Research [MOP77548]; the Canada Foundation for Innovation; and the Jewish Rehabilitation Hospital Foundation. JB received a doctoral training award from the Canadian Institute of Health Research and the Heart and Stroke Foundation of Canada.

\section{References}

1. Gibson JJ (1950) Perception of the Visual World: Houghton Mifflin.

2. Sarre G, Berard J, Fung J, Lamontagne A (2008) Steering behaviour can be modulated by different optic flows during walking. Neurosci Lett 436: 96-101.

3. Warren WH Jr, Kay BA, Zosh WD, Duchon AP, Sahuc S (2001) Optic flow is used to control human walking. Nat Neurosci 4: 213-236.

4. Berard J, Fung J, Lamontagne A (2011) Evidence for the use of rotational optic flow cues for locomotor steering in healthy older adults. J Neurophysiol 106: 1089-1096.

5. Rowe F, Brand D, Jackson CA, Price A, Walker L, et al. (2009) Visual impairment following stroke: do stroke patients require vision assessment? Age Ageing 38: 188-193.

6. Lamontagne A, Fung J (2009) Gaze and postural reorientation in the contro of locomotor steering after stroke. Neurorehabil Neural Repair 23: 256-266.

7. Imai T, Moore ST, Raphan T, Cohen B (2001) Interaction of the body, head, and eyes during walking and turning. Exp Brain Res 136: 1-18.

8. Vaina LM, Sikoglu EM, Soloviev S, LeMay M, Squatrito S, et al. (2010) Functional and anatomical profile of visual motion impairments in stroke patients correlate with fMRI in normal subjects. J Neuropsychol 4: 121-145.

9. Billino J, Braun DI, Böhm KD, Bremmer F, Gegenfurtner KR (2009) Cortical networks for motion processing: effects of focal brain lesions on perception of different motion types. Neuropsychologia 47: 2133-2144.

10. Lamontagne A, Fung J, McFadyen B, Faubert J (2007) Modulation of walking speed by changing optic flow in persons with stroke. J Neuroeng Rehabil 4: 22.

11. Lamontagne A, Fung J, McFadyen B, Faubert J, Paquette C (2010) Stroke affects locomotor steering responses to changing optic flow directions. Neurorehabil Neural Repair 24: 457-468.

12. Folstein MF, Folstein SE, McHugh PR (1975) "Mini-mental state". A practical method for grading the cognitive state of patients for the clinician. J Psychiatr Res 12: 189-198.

13. Gowland C, Stratford P, Ward M, Moreland J, Torresin W, et al. (1993). Measuring physical impairment and disability with the Chedoke-McMaster Stroke Assessment. Stroke 24: 58-63.

14. Bisiach E, Bulgarelli C, Sterzi R, Vallar G (1983) Line bisection and cognitive plasticity of unilateral neglect of space. Brain Cogn 2: 32-38.

15. Nasreddine ZS, Phillips NA, Bédirian V, Charbonneau S, Whitehead V, et al (2005) The Montreal Cognitive Assessment, MoCA: a brief screening tool for mild cognitive impairment. J Am Geriatr Soc 53: 695-699.

16. Strauss E, Sherman E, Spreen O (2006) A Compendium of Neuropsychological Tests: Administration, Norms, and Commentary (3rdedn), New York: Oxford University Press.

17. Tombaugh TN (2004) Trail Making Test A and B: normative data stratified by age and education. Arch Clin Neuropsychol 19: 203-214.

This article was originally published in a special issue, Stroke:

Cerebrovascular accident handled by Editor(s). Dr. David Della Morte,

University of Miami, USA
18. Walker ML, Austin AG, Banke GM, Foxx SR, Gaetano L, et al. (2007) Reference group data for the functional gait assessment. Phys Ther 87: 1468-1477.

19. Barton JJ, Sharpe JA, Raymond JE (1996) Directional defects in pursuit and motion perception in humans with unilateral cerebral lesions. Brain 119: 15351550.

20. Schenk T, Zihl J (1997) Visual motion perception after brain damage: I. Deficits in global motion perception. Neuropsychologia 35: 1289-1297.

21. Zihl J, von Cramon D, Mai N, Schmid C (1991) Disturbance of movemen vision after bilateral posterior brain damage. Further evidence and follow up observations. Brain 114: 2235-2252.

22. Harris JM, Bonas W (2002) Optic flow and scene structure do not always contribute to the control of human walking. Vision Res 42: 1619-1626.

23. Rushton SK, Harris JM, Lloyd MR, Wann JP (1998) Guidance of locomotion on foot uses perceived target location rather than optic flow. Curr Biol 8: 1191 1194.

24. Turton AJ, Dewar SJ, Lievesley A, O'Leary K, Gabb J, et al. (2009) Walking and wheelchair navigation in patients with left visual neglect. Neuropsychol Rehabil 19: $274-290$

25. Berti A, Smania N, Rabuffetti M, Ferrarin M, Spinazzola L, et al. (2002) Coding of far and near space during walking in neglect patients. Neuropsychology 16 390-399.

26. Robertson IH, Tegner R, Goodrich SJ, Wilson C (1994) Walking trajectory and hand movements in unilateral left neglect: a vestibular hypothesis. Neuropsychologia 32: 1495-1502.

27. Tromp E, Dinkla A, Mulder T (1995) Walking through doorways: An analysis of navigation skills in patients with neglect. Neuropsychol Rehabil 5: 319-331.

28. Huitema RB, Brouwer WH, Hof AL, Dekker R, Mulder T, et al. (2006) Walking trajectory in neglect patients. Gait Posture 23: 200-205.

29. Fordell H, Bodin K, Bucht G, Malm J (2011) A virtual reality test battery for assessment and screening of spatial neglect. Acta Neurol Scand 123: 167-174

30. Cowey A, Small M, Ellis S (1999) No abrupt change in visual hemineglect from near to far space. Neuropsychologia 37: 1- 6.

31. Keller I, Schindler I, Kerkhoff G, von Rosen F, Golz D (2005) Visuospatia neglect in near and far space: dissociation between line bisection and letter cancellation. Neuropsychologia 43: 724-731.

32. Dvorkin AY, Bogey RA, Harvey RL, Patton JL (2011) Mapping the Neglected Space: Gradients of Detection Revealed by Virtual Reality. Neurorehabil Neural Repair 26: 120-131.

33. Karnath HO, Rorden C (2011) The anatomy of spatial neglect. Neuropsychologia.

34. Fruhmann Berger M, Johannsen L, Karnath HO (2009) Subcortical neglect is not always a transient phenomenon: evidence from a 1-year follow-up study. J Clin Exp Neuropsychol 31: 617-623. 